\title{
Comparison of efficacy between low-fluence and half-dose verteporfin photodynamic therapy for chronic central serous chorioretinopathy
}

This article was published in the following Dove Press journal:

Clinical Ophthalmology

5 April 2014

Number of times this article has been viewed

\section{Zeynep Alkin' \\ Irfan Perente \\ Abdullah Ozkaya' \\ Dilek Alp' \\ Alper Agca' \\ Ebru Demet Aygit \\ Selcuk Korkmaz ${ }^{2}$ \\ Ahmet Taylan Yazici' \\ Ahmet Demirok' \\ 'Beyoglu Eye Training and Research Hospital, Istanbul, Turkey; ${ }^{2}$ Hacettepe University Faculty of Science, Department of Statistics, Ankara, Turkey}

Correspondence: Zeynep Alkin Beyoglu Eye Training and Research Hospital, Bereketzade Cami Sok No 2 Beyoglu, İstanbul, Turkey Tel +905052703658

Fax +90 21 22526109 Email zeynepalkin@gmail.com
Purpose: To compare the efficacy of low-fluence photodynamic therapy (PDT) and PDT with half-dose verteporfin in chronic central serous chorioretinopathy (CSC).

Patients and methods: The medical records of 64 eyes from 60 patients with chronic CSC were retrospectively reviewed; 36 eyes received low-fluence PDT $\left(25 \mathrm{~J} / \mathrm{cm}^{2}\right)$ and 28 eyes received half-dose verteporfin PDT $\left(3 \mathrm{mg} / \mathrm{m}^{2}\right)$. The primary outcome measure was the proportion of eyes with complete resolution of subretinal fluid. Secondary outcome measures were the changes in best corrected visual acuity (BCVA) and central foveal thickness, and the proportion of eyes that showed an increase of $\geq 5$ letters in BCVA at the last visit.

Results: The mean follow-up period was $12.5 \pm 4.3$ months and $13.1 \pm 4$ months in the low-fluence group and half-dose group, respectively $(P=0.568)$. Thirty-three eyes $(91.6 \%)$ in the low-fluence group and 26 eyes $(92.8 \%)$ in the half-dose verteporfin group showed complete resolution of subretinal fluid $(P=0.703)$. BCVA increased by a mean of 7.4 letters and 4.8 letters in the low-fluence group and half-dose group, respectively $(P=0.336)$. Seventeen eyes $(52.8 \%)$ in the low-fluence group and 14 eyes (50\%) in the half-dose group experienced a gain of $\geq 5$ letters in BCVA $(P=0.825)$. In the low-fluence and half-dose verteporfin group, the mean baseline central foveal thickness was $351 \pm 90 \mu \mathrm{m}$ and $341 \pm 96 \mu \mathrm{m}$, and significantly decreased to $188 \pm 61 \mu \mathrm{m}$ and $181 \pm 47 \mu \mathrm{m}$, respectively $(P<0.01)$.

Conclusion: Both treatments resulted in complete subretinal fluid resolution in most of the eyes, with significantly better visual acuity outcomes compared to baseline at the last visit.

Keywords: low-fluence, half-dose verteporfin, photodynamic therapy, central serous chorioretinopathy

\section{Introduction}

Chronic central serous chorioretinopathy (CSC) is a disease characterized by long standing serous neurosensory retinal detachment in the central macular region., Chronic disease can be associated with widespread alteration of the retinal pigment epithelium (RPE), neuroretinal degeneration, and secondary choroidal neovascularization, leading to eventual visual acuity loss related to persistent or recurrent serous retinal detachment. ${ }^{3-5}$ Photodynamic therapy (PDT) has been shown to enhance the absorption of subretinal fluid by reducing choroidal vascular hyperpermeability. ${ }^{6-8}$ However, using conventional verteporfin dosage and laser fluence of PDT in the treatment of chronic CSC is associated with choriocapillaris hypoperfusion, RPE atrophy, and secondary choroidal neovascularization, resulting in decreased vision..$^{9,10}$ Due to treatment related complications, the parameters for standard PDT have been reconsidered, either by reducing the dose of verteporfin or by reducing the fluence rate 
to minimize retinal damage and obtain sufficient treatment effects on the choroidal vasculature. In previous reports, modified PDT protocols, with reduced verteporfin dosages and fluence rates, have been shown to induce the resolution of subretinal fluid and provide significant improvement in vision in chronic CSC with low complication rates. ${ }^{11-14}$ However, to date, no study has investigated whether one treatment method results in better visual acuity and anatomical outcomes in the treatment of chronic CSC than other PDT protocols. In the present study, we investigated the efficacy and the rate of side effects of low-fluence PDT for the treatment of chronic CSC compared to PDT with half-dose verteporfin.

\section{Patients and methods}

\section{Study design}

We retrospectively reviewed the medical records of 60 patients with chronic CSC who underwent PDT between June 2005 and July 2011 at Beyoglu Eye Training and Research Hospital, Turkey. Approval for data collection and analysis was obtained from the ethics committee of the hospital, and all of the patients provided informed consent. The methodology of the study was designed in accordance with the tenets of the Declaration of Helsinki.

\section{Study population}

The inclusion criteria for the study were as follows: 1) best corrected visual acuity (BCVA) $\geq 50$ Early Treatment Diabetic Retinopathy Study ${ }^{15}$ (ETDRS) letters (Snellen equivalent 20/200); 2) persistent or progressive symptoms, such as visual disturbances or distortions or metamorphopsia consistent with CSC for more than 3 months; 3) retina pigment epithelium decompensation with focal or diffuse subfoveal leakage on fluorescein angiography (FA); 4) abnormally dilated choroidal vasculature and choroidal vascular hyperpermeability appearing as hyperfluorescence on indocyanine green angiography (ICGA); and 5 ) presence of subretinal fluid in the fovea with or without serous pigment epithelial detachment on optical coherence tomography (OCT).

The exclusion criteria were as follows: 1) previous focal laser, PDT, or intraocular injection for treatment of CSC; 2) evidence of choroidal neovascular membrane (CNV), polypoidal choroidal vasculopathy, or other macular pathologies other than CSC, such as diabetic retinopathy, posterior uveitis, and retinal vein occlusion; 3) any systemic disease causing elevated blood steroid levels, including exogenous corticosteroid treatment, or Cushing's disease; 4) pregnancy; and 5) systemic contraindications for PDT.

\section{Diagnostic evaluation and follow-up examinations}

Diagnosis of chronic CSC was based on the findings on fundoscopy, FA, ICGA (Heidelberg Retinal Angiograph; Heidelberg Engineering, Heidelberg, Germany), and OCT (Stratus OCT 3000; Carl Zeiss Meditec, Dublin, CA, USA). A complete ophthalmic examination, including BCVA measurement using ETDRS chart at 4 meters, applanation tonometry, and fundus examination using a 90 diopter indirect lens after mydriasis, was performed at baseline, at 1 month, at 3 months, and at 3-month intervals thereafter. Special diagnostic evaluations, including FA, ICGA, and Stratus OCT examination, were performed at baseline to confirm the diagnosis and to guide the treatment. Then, OCT images were obtained at every control visit to determine the outcome after PDT. Central foveal thickness (CFT) was measured using the fast macular thickness program within a scan time of 1.9 seconds. The device scans six evenly spaced $6 \mathrm{~mm}$ radial lines, consisting of $128 \mathrm{~A}$ scans per line that intersect at the fovea (total of 768 sampled points) ${ }^{15} \mathrm{CFT}$, defined as the mean thickness of the neurosensory retina in a central $1 \mathrm{~mm}$ diameter area, was computed using OCT mapping software packaged with the device. Additional FA and ICGA were performed during follow-up in eyes with persistent or recurrent subretinal fluid.

\section{Treatment protocol}

The patients with chronic CSC received either low-fluence PDT $\left(25 \mathrm{~J} / \mathrm{cm}^{2}\right)$ or half-dose PDT with verteporfin $\left(3 \mathrm{mg} / \mathrm{m}^{2}\right)$. Low-fluence PDT was performed with a $6 \mathrm{mg} / \mathrm{m}^{2}$ dose of verteporfin but with a reduced fluence of $25 \mathrm{~J} / \mathrm{cm}^{2}$ over a duration of 83 seconds. Half-dose PDT was administered with half of the standard dose of verteporfin $\left(3 \mathrm{mg} / \mathrm{m}^{2}\right)$, and the duration and power of the laser application remained unchanged, with a total light energy of $50 \mathrm{~J} / \mathrm{cm}^{2}$ for 83 seconds. In both groups, the treatment spot size was confined to the area of choroidal abnormality, including the dilated and congested choroidal vessels, and to the area with sub-RPE leakage in the macula, according to findings by ICGA. Both eyes were treated within the same session if they had subretinal fluid in the fovea at presentation. After treatment, protective spectacles were provided, and the patients were instructed to avoid direct sunlight or strong light for 3 days.

\section{Outcome parameters}

The primary outcome measure of the study was the proportion of eyes with complete resolution of subretinal fluid at the last follow-up. The secondary outcomes were the 
changes in mean BCVA and mean CFT and the proportion of eyes that showed increase of $\geq 5$ letters in BCVA at the last follow-up.

\section{Statistical analysis}

Numerical data were summarized using means and standard deviations, while categorical data were summarized using frequencies and percentages. Categorical variables were analyzed using the chi-square test or Fisher's exact test. Continuous variables were compared between the groups, and pre- and posttreatment values were compared within the groups using the nonparametric Mann-Whitney $U$ test and Wilcoxon signed rank test where appropriate. The statistical evaluation was performed using SPSS software (version 16.0; SPSS Inc., Chicago, IL, USA). A $P$-value of less than 0.05 was considered to be statistically significant.

\section{Results}

A total of 64 eyes from 60 patients with chronic CSC, of which 36 received low-fluence PDT and 28 received PDT with halfdose verteporfin, were recruited. The baseline characteristics of both groups are shown in the Table 1. All the patients included in this study had leakage from choroidal vessels adjacent to the fovea and therefore had received treatment including the fovea. At baseline, there were no significant differences between the two groups. The mean follow-up

Table I Baseline characteristics, visual acuity, central foveal thickness, photodynamic therapy spot size, and mean follow-up period in low-fluence and half-dose groups

\begin{tabular}{|c|c|c|c|}
\hline Variables & $\begin{array}{l}\text { Low-fluence } \\
\text { group } \\
\text { ( } 34 \text { patients, } \\
36 \text { eyes) }\end{array}$ & $\begin{array}{l}\text { Half-dose } \\
\text { group } \\
\text { ( } 26 \text { patients, } \\
28 \text { eyes) }\end{array}$ & $P$-value \\
\hline $\begin{array}{l}\text { Mean age } \pm \text { SD (years)* } \\
\text { (range) }\end{array}$ & $\begin{array}{l}45.1 \pm 7.6 \\
(29-60)\end{array}$ & $\begin{array}{l}44.4 \pm 9.8 \\
(27-66)\end{array}$ & 0.758 \\
\hline Sex (male/female $)^{\dagger}$ & $28 / 6$ & $21 / 5$ & 0.875 \\
\hline $\begin{array}{l}\text { Mean duration of } \\
\text { symptoms } \pm S D \text { (months)* } \\
\text { (range) }\end{array}$ & $\begin{array}{l}12.4 \pm 10.1 \\
(3-40)\end{array}$ & $\begin{array}{l}17.1 \pm 19.2 \\
(3-80)\end{array}$ & 0.213 \\
\hline $\begin{array}{l}\text { Number of eyes } \\
\text { with PED }(\%)^{\ddagger}\end{array}$ & $4(11.1 \%)$ & $3(10.7 \%)$ & 0.96 \\
\hline $\begin{array}{l}\text { Mean PDT spot } \\
\text { size } \pm \text { SD }(\mu \mathrm{m})^{*} \\
(\text { range) }\end{array}$ & $\begin{array}{l}2,662 \pm 819 \\
(1,000-4,500)\end{array}$ & $\begin{array}{l}2,405 \pm 739 \\
(1,290-4,000)\end{array}$ & 0.302 \\
\hline $\begin{array}{l}\text { Mean duration of } \\
\text { follow-up } \pm \text { SD (months)* } \\
\text { (range) }\end{array}$ & $\begin{array}{l}12.5 \pm 4.3 \\
(6-18)\end{array}$ & $\begin{array}{l}13.1 \pm 4 \\
(6-18)\end{array}$ & 0.568 \\
\hline
\end{tabular}

Notes: *Denotes Mann-Whitney $U$ test; ${ }^{\dagger}$ denotes chi-square test; and ${ }^{\ddagger}$ denotes Fisher's exact test.

Abbreviations: PDT, photodynamic therapy; PED, pigment epithelium detachment; $\mathrm{SD}$, standard deviation. period was $12.5 \pm 4.3$ months (range, 6-18 months) in the low-fluence group and 13.1 \pm 4 months (range, 6-18 months) in the half-dose group $(P=0.568)$. Thirty-one of the 36 eyes in the low-fluence group and 25 of the 28 eyes in the half-dose verteporfin group showed no subretinal fluid (SRF) on OCT at 3 months. Of the remaining two eyes (5.5\%) in the lowfluence group and one eye (3.5\%) in the half-dose verteporfin group, these were successfully retreated with PDT with the same protocol as in the first treatment. Three eyes in the lowfluence group and two eyes in the half-dose verteporfin group were given no treatment because the patients were unwilling to receive any further treatment. Overall complete resolution of SRF was achieved in 33 eyes (91.6\%) in the low-fluence group, which was not clinically significant from the half-dose verteporfin group (26 eyes, 92.8\%) $(P=0.703)$

\section{Visual acuity outcomes}

The BCVA at baseline, post-PDT 1 month, post-PDT 3 months, post-PDT 6 months, and at the last visit are listed in Table 2 and are shown in Figure 1. BCVA at post-PDT 1 month, 3 months, 6 months, and at the last visit were all significantly improved compared with the baseline BCVA in both groups $(P<0.01$ for each). When the two groups were compared, the amount of improvement of visual acuity did not significantly differ between the two groups at any of the periods $(P=0.312,0.606,0.088$, and 0.311 , respectively).

Table 2 Changes in best corrected visual acuity in the lowfluence group and half-dose verteporfin group after photodynamic therapy

\begin{tabular}{|c|c|c|c|c|}
\hline $\begin{array}{l}\text { Time } \\
\text { point }\end{array}$ & $\begin{array}{l}\text { BCVA } \\
\text { (ETDRS } \\
\text { letters) }\end{array}$ & $\begin{array}{l}\text { Low-fluence } \\
\text { group } \\
\text { ( } 34 \text { patients, } \\
36 \text { eyes) }\end{array}$ & $\begin{array}{l}\text { Half-dose } \\
\text { group } \\
\text { ( } 26 \text { patients, } \\
28 \text { eyes) }\end{array}$ & $P$-value* \\
\hline \multirow[t]{2}{*}{ Baseline } & Mean & 82.1 & 85.6 & - \\
\hline & $\begin{array}{l}\text { Min, median, } \\
\max \end{array}$ & $50,83,97$ & $51,87,97$ & 0.257 \\
\hline \multirow[t]{2}{*}{ I month } & Mean & 88.8 & 90.6 & - \\
\hline & $\begin{array}{l}\text { Min, median, } \\
\max \end{array}$ & $65,89,99$ & $50,92,100$ & 0.312 \\
\hline \multirow[t]{2}{*}{3 months } & Mean & 88.2 & 90.7 & - \\
\hline & $\begin{array}{l}\text { Min, median, } \\
\max \end{array}$ & $57,93,99$ & $50,94,99$ & 0.606 \\
\hline \multirow[t]{2}{*}{6 months } & Mean & 90 & 90.2 & - \\
\hline & $\begin{array}{l}\text { Min, median, } \\
\max \end{array}$ & $53,88,99$ & $55,95,99$ & 0.088 \\
\hline \multirow[t]{2}{*}{ Last visit } & Mean & 89.6 & 90.5 & - \\
\hline & $\begin{array}{l}\text { Min, median, } \\
\max \end{array}$ & $56,92,100$ & $55,95,100$ & 0.311 \\
\hline
\end{tabular}

Note: ${ }^{*} P$-value based on Mann-Whitney $U$ test of median difference equal to zero. Abbreviations: BCVA, best corrected visual acuity; ETDRS, Early Treatment Diabetic Retinopathy Study; max, maximum; min, minimum. 


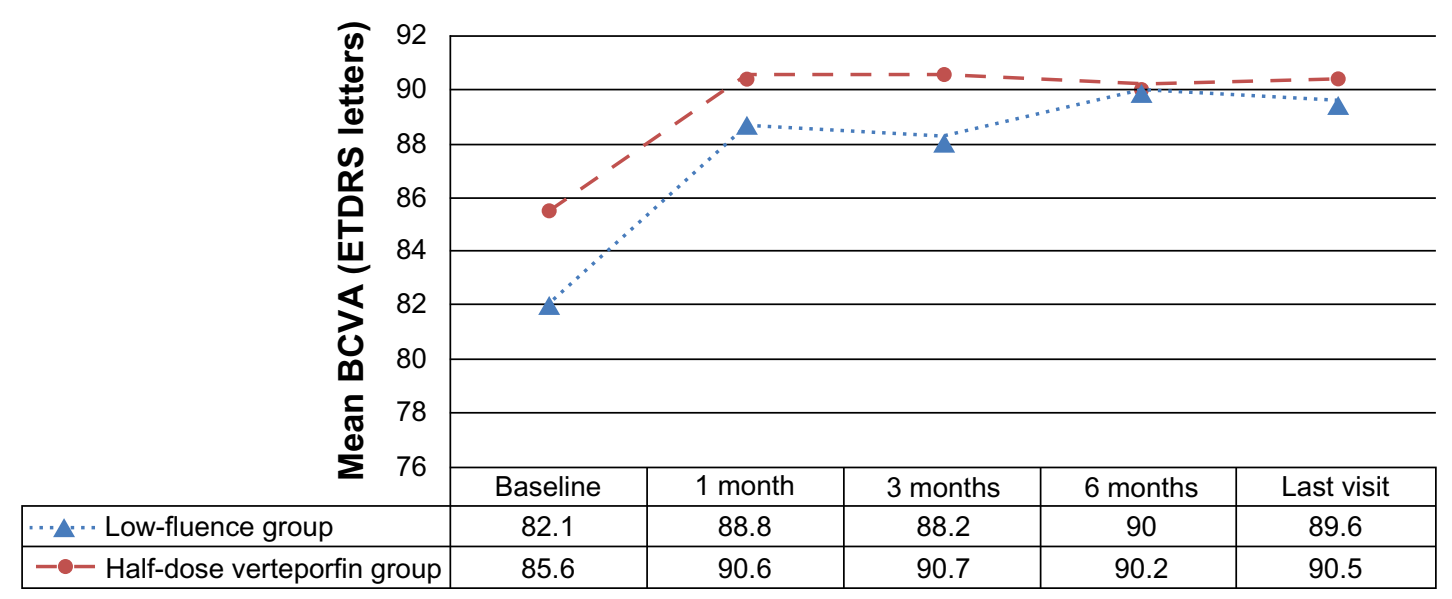

Figure I Time course of the mean best corrected visual acuity of eyes with chronic central serous chorioretinopathy that underwent low-fluence photodynamic therapy and photodynamic therapy with half-dose verteporfin.

Abbreviations: BCVA, best corrected visual acuity; ETDRS, Early Treatment Diabetic Retinopathy Study

BCVA increased by a mean of 7.4 letters in the lowfluence group and 4.8 letters in the half-dose group at the last visit $(P=0.336)$. A total of 17 (52.8\%) low-fluence treated eyes experienced a gain of $\geq 5$ letters in BCVA compared with 14 $(50 \%)$ of half-dose verteporfin treated eyes $(P=0.825)$. Two eyes $(5.6 \%)$ in the low-fluence group and one eye $(3.6 \%)$ in the half-dose verteporfin group had a loss of $\geq 5$ letters due to the persistent subretinal fluid at the macula.

\section{OCT outcomes}

The mean CFT measured with OCT at baseline, post-PDT 1 month, post-PDT 3 months, post-PDT 6 months, and at the last visit are listed in Table 3 and are shown in Figure 2. The mean CFT at post-PDT 1 month, post-PDT 3 months, postPDT 6 months, and at the last visit were all significantly thinner than that at baseline in both groups ( $P<0.01$ for each). When the two groups were compared, the amount of change of CFT did not significantly differ between the two groups at any of the periods ( $P=0.802,0.846,0.758$, and 0.943 , respectively).

Examples of the changes in ICGA and OCT of the patients in the low-fluence and half-dose verteporfin groups are displayed in Figures 3 and 4.

No patients in either group developed any systemic side effects related to verteporfin treatment, including infusion site complications and lower back pain. During the follow-up period, neither RPE atrophy nor the development of choroidal neovascular membranes was observed in the eyes in either group.

\section{Discussion}

This retrospective study comparing the efficacy of lowfluence PDT and PDT with half-dose verteporfin for chronic
CSC revealed similar improvements in visual acuity and foveal thinning. Both treatment strategies resulted in a higher proportion of eyes with absence of subretinal fluid at the last visit.

Currently, there is no standard therapy for chronic CSC. A large number of studies have reported favorable results, with significant increases in BCVA and improvement of serous macular detachments, with PDT in chronic CSC since its first report in 2003 by Cardillo Piccolino et al. ${ }^{7}$ Unfortunately, PDT also has potential drawbacks, including damage to the cho-

Table 3 Changes in central foveal thickness in the low-fluence group and half-dose verteporfin group after photodynamic therapy

\begin{tabular}{|c|c|c|c|c|}
\hline $\begin{array}{l}\text { Time } \\
\text { point }\end{array}$ & CFT $(\mu \mathrm{m})$ & $\begin{array}{l}\text { Low-fluence } \\
\text { group } \\
\text { ( } 34 \text { patients, } \\
36 \text { eyes) }\end{array}$ & $\begin{array}{l}\text { Half-dose } \\
\text { group } \\
\text { ( } 26 \text { patients, } \\
28 \text { eyes) }\end{array}$ & P-value* \\
\hline \multirow[t]{2}{*}{ Baseline } & Mean \pm SD & $35 I \pm 90$ & $341 \pm 96$ & - \\
\hline & $\begin{array}{l}\text { Min, median, } \\
\max \end{array}$ & $208,367,640$ & $210,342,525$ & 0.685 \\
\hline \multirow[t]{2}{*}{ I month } & Mean \pm SD & $194 \pm 59$ & $180 \pm 43$ & - \\
\hline & $\begin{array}{l}\text { Min, median, } \\
\max \end{array}$ & $110,215,377$ & $119,192,312$ & 0.802 \\
\hline \multirow[t]{2}{*}{3 months } & Mean \pm SD & $190 \pm 65$ & $178 \pm 48$ & - \\
\hline & $\begin{array}{l}\text { Min, median, } \\
\max \end{array}$ & III, 208, 4I8 & $126,189,333$ & 0.846 \\
\hline \multirow[t]{2}{*}{6 months } & Mean \pm SD & $|8| \pm 4 \mid$ & $176 \pm 50$ & - \\
\hline & $\begin{array}{l}\text { Min, median, } \\
\max \end{array}$ & $103,215,398$ & $130,187,325$ & 0.758 \\
\hline \multirow[t]{2}{*}{ Last visit } & Mean \pm SD & $|88 \pm 6|$ & $|8| \pm 47$ & - \\
\hline & $\begin{array}{l}\text { Min, median, } \\
\max \end{array}$ & $108,204,4 \mid I$ & $119,190,306$ & 0.943 \\
\hline
\end{tabular}

Note: $* P$-value based on Wilcoxon signed rank test of median difference equal to zero.

Abbreviations: BCVA, best corrected visual acuity; CFT, central foveal thickness; ETDRS, Early Treatment Diabetic Retinopathy Study; max, maximum; min, minimum; $\mathrm{SD}$, standard deviation. 


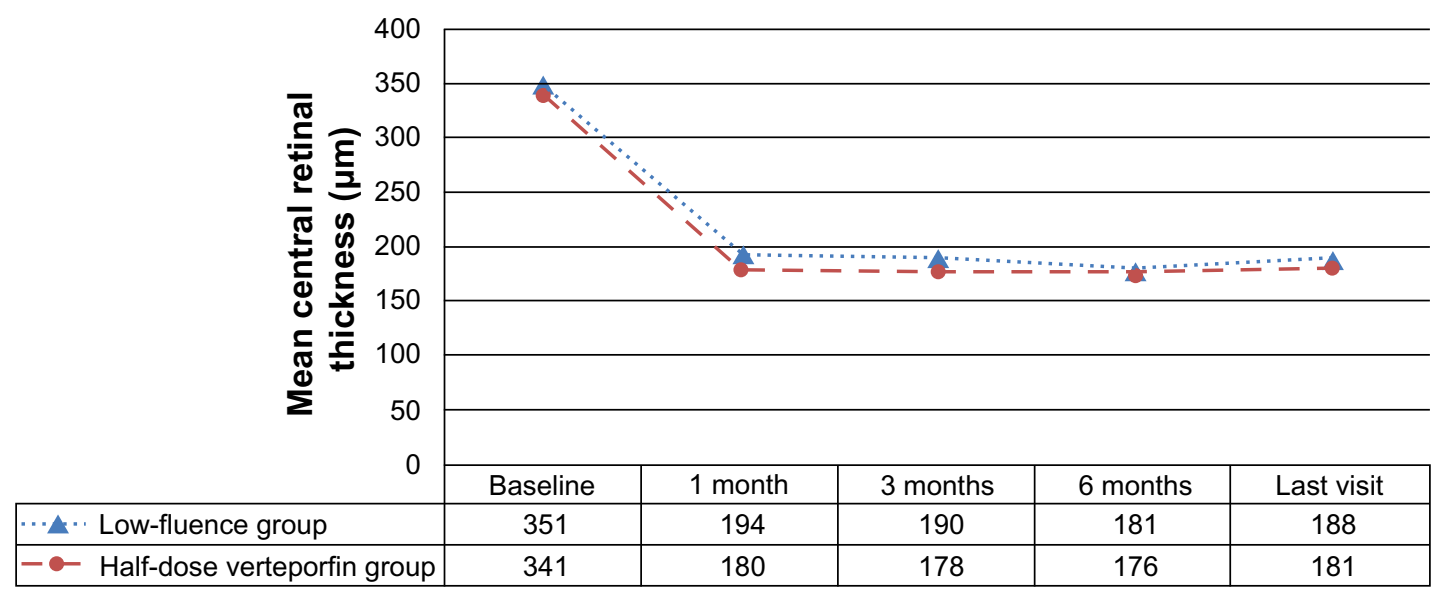

Figure 2 Time course of the mean central foveal thickness on optical coherence tomography of the eyes with chronic central serous chorioretinopathy which underwent low-fluence photodynamic therapy and photodynamic therapy with half-dose verteporfin.

riocapillaris, permanent RPE atrophy, and the development of $\mathrm{CNV}$ related to the hypoxic damage caused by choriocapillaris occlusion at the site of PDT. ${ }^{9,10}$ These potential side effects may be important considerations that restrict the use of PDT using standard parameters for chronic CSC because such patients generally have good visual acuity at presentation. It has been proposed that PDT parameters could be modified to achieve decreased choroidal vascular hyperpermeability without adverse effects on the choroidal vessels and RPE. ${ }^{12,17}$ Using half the dose of verteporfin, Chan et al showed complete resolution of serous detachment in $89.6 \%$ of eyes at 12 months, without systemic or ocular side effects, in an uncontrolled series of

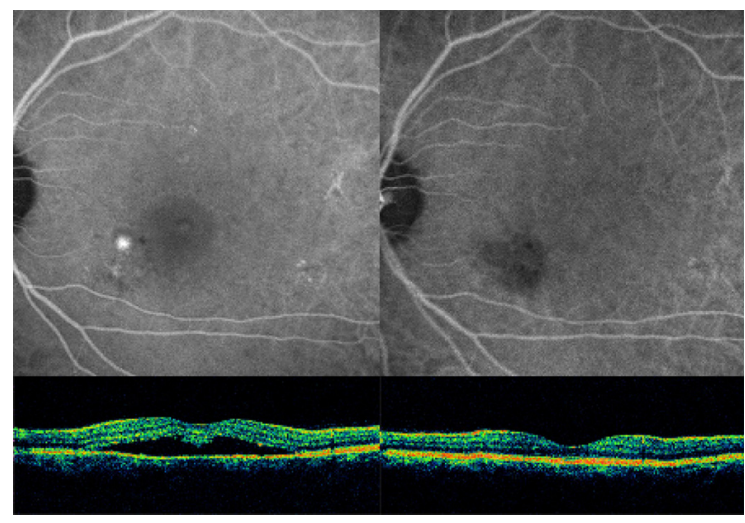

Figure 3 Images from the left eye of a patient with chronic central serous chorioretinopathy.

Notes: The patient was treated with low-fluence photodynamic therapy at baseline (left column) and after therapy (right column). Late phase indocyanine green angiography showed perifoveal choroidal focal leaks consistent with central serous chorioretinopathy (top left). There was no hyperfluorescence leakage, with a minimal perfusion defect in the choriocapillaris with late phase indocyanine green angiography at 6 months of therapy (top right). Optical coherence tomography showed the presence of subretinal fluid and elongation of the photoreceptor outer segments (central foveal thickness; $386 \mu \mathrm{m}$ ) (bottom left). Optical coherence tomography imaging confirmed the complete resolution of the subretinal fluid at 6 months (central foveal thickness; $170 \mu \mathrm{m}$ ) (bottom right). cases with chronic CSC. ${ }^{11}$ In the study by Nicolò et al, complete resolution of subretinal fluid was obtained in $92.1 \%$ of eyes at the last follow-up of 14 months after PDT with half-dose verteporfin. ${ }^{18}$ The mean Logarithm of the Minimum Angle of Resolution (logMAR) BCVA improved significantly, from 0.31 to 0.20 at 3 months, and showed further improvement until month 12 in the aforementioned study conducted by Chan et al. ${ }^{11}$ Additionally, the fluence rate could be modified. Reibaldi et al showed that at 12 months, low-fluence PDT resulted in BCVA improvement of $0.31 \log$ MAR units and resolution of subretinal fluid in $91 \%$ of eyes with a lower grade of hypoperfusion of the choroid. ${ }^{13}$ The authors proposed that

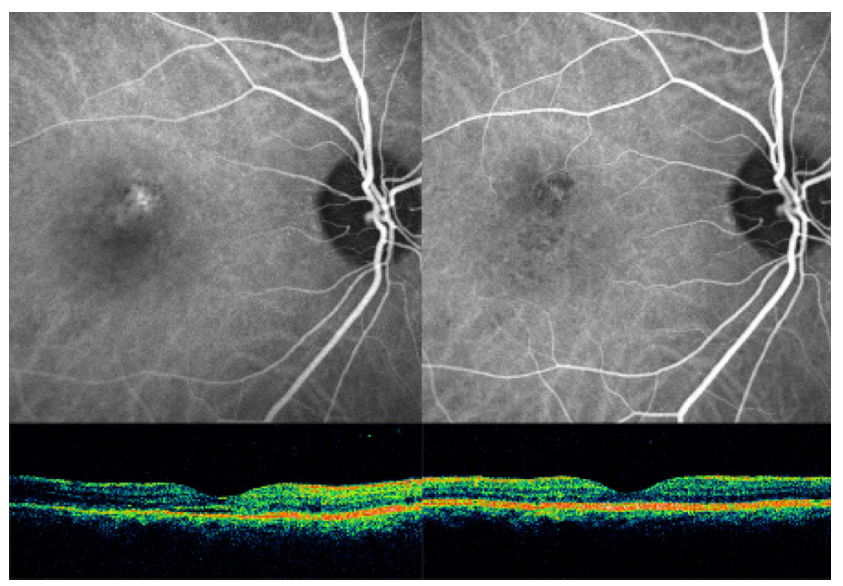

Figure 4 Images from the right eye of a patient with chronic central serous chorioretinopathy treated with photodynamic therapy with half-dose verteporfin, at baseline (left column) and after therapy (right column).

Notes: Middle phase indocyanine green angiography showed central choroidal vascular hyperpermeability (top left) and no leakage at the site of photodynamic therapy application (top right). Optical coherence tomography revealed a shallow submacular neurosensory retinal detachment (central foveal thickness; $169 \mu \mathrm{m}$ ) (bottom left). Optical coherence tomography demonstrated the complete resolution of subretinal fluid, with minimal thinning of the neuroretina (central foveal thickness: $142 \mu \mathrm{m})$ (bottom right). 
reducing the fluence rate may prevent excessive activation of verteporfin and could restrict the generation of singlet oxygen by activated verteporfin in the treated area. They observed a trend toward further BCVA improvement in the low-fluence group but not in the standard-fluence group.

Overall, our study demonstrated that at the last visit, lowfluence and half-dose verteporfin PDT resulted in complete resolution of subretinal fluid in $91.6 \%$ and $92.8 \%$ of eyes at the last visit, respectively, without significant differences between the two treatments, which confirms previous reports using both treatment strategies. The mean BCVA of patients in both treatment groups at all follow-up visits was significantly better when compared to baseline. The mean BCVA improvement of 7.4 letters and 4.8 letters in the low-fluence group and half-dose verteporfin group, respectively, showed no significant difference between the groups.

This study has its shortcomings in that it was not a prospective, randomized clinical study, the follow-up duration varied between the cases, and it included a limited number of patients. Additionally, follow-up ICGA was performed only as needed, not on a regular basis, after treatment. The long-term effects of decreased choroidal perfusion on visual function were not possible to evaluate in this study, and a further investigation is needed to determine changes in choriocapillaris perfusion with time in the areas to which treatment is applied. Further research should also determine whether the damage to normal choroidal vasculature caused by PDT can be reduced, possibly by combining low-fluence PDT with a reduced dose of verteporfin.

In conclusion, the results of this study suggest that both low-fluence PDT and PDT with half-dose verteporfin may be beneficial in treating chronic CSC. In this comparison study, both treatments showed similar resolution rates of subretinal fluid in chronic CSC with promising functional outcomes over the long-term.

\section{Disclosure}

The authors have no proprietary or commercial interest in any materials discussed in this article. No financial or material support has been used for this work. The authors disclose that there is no funding or financial support in connection with this article.

Clinical Ophthalmology

\section{Publish your work in this journal}

Clinical Ophthalmology is an international, peer-reviewed journal covering all subspecialties within ophthalmology. Key topics include: Optometry; Visual science; Pharmacology and drug therapy in eye diseases; Basic Sciences; Primary and Secondary eye care; Patient Safety and Quality of Care Improvements. This journal is indexed on Submit your manuscript here: http://www.dovepress.com/clinical-ophthalmology-journal

\section{References}

1. Prunte C, Flammer J. Choroidal capillary and venous congestion in central serous chorioretinopathy. Am J Ophthalmol. 1996;121(1):26-34.

2. Piccolino FC, Borgia L. Central serous chorioretinopathy and indocyanine green angiography. Retina. 1994;14(3):231-242.

3. Loo RH, Scott IU, Flynn HW Jr, et al. Factors associated with reduced visual acuity during long-term follow-up of patients with idiopathic central serous chorioretinopathy. Retina. 2002;22(1):19-24.

4. Moon JW, Yu HG, Kim TW, Kim HC, Chung H. Prognostic factors related to photodynamic therapy for central serous chorioretinopathy. Graefes Arch Clin Exp Ophthalmol. 2009;247(10):1315-1323.

5. Piccolino FC, de la Longrais RR, Ravera G, et al. The foveal photoreceptor layer and visual acuity loss in central serous chorioretinopathy. Am J Ophthalmol. 2005;139(1):87-99.

6. Yannuzzi LA, Slakter JS, Gross NE, et al. Indocyanine green angiography-guided photodynamic therapy for treatment of chronic central serous chorioretinopathy: a pilot study. Retina. 2003;23(3): 288-298.

7. Cardillo Piccolino F, Eandi CM, Ventre L, Rigault de la Longrais RC, Grignolo FM. Photodynamic therapy for chronic central serous chorioretinopathy. Retina. 2003;23(6):752-763.

8. Silva RM, Ruiz-Moreno JM, Gomez-Ulla F, et al. Photodynamic therapy for chronic central serous chorioretinopathy: a 4-year follow-up study. Retina. 2013;33(2):309-315.

9. Chan WM, Lam DS, Lai TY, Tam BS, Liu DT, Chan CK. Choroidal vascular remodelling in central serous chorioretinopathy after indocyanine green guided photodynamic therapy with verteporfin: a novel treatment at the primary disease level. Br J Ophthalmol. 2003;87(12):1453-1458.

10. Colucciello M. Choroidal neovascularization complication photodynamic therapy for central serous retinopathy. Retina. 2006;26(2):239-242.

11. Chan WM, Lai TY, Lai RY, Tang EW, Liu DT, Lam DS. Safety enhanced photodynamic therapy for chronic central serous chorioretinopathy: one year results of a prospective study. Retina. 2008;28(1):85-93.

12. Lai TY, Chan WM, Li H, Lai RY, Liu DT, Lam DS. Safety enhanced photodynamic therapy with half dose verteporfin for chronic central serous chorioretinopathy: a short term pilot study. Br J Ophthalmol. 2006;90:869-874.

13. Reibaldi M, Cardascia N, Longo A, et al. Standard-fluence versus low-fluence photodynamic therapy in chronic central serous chorioretinopathy: a nonrandomized clinical trial. Am J Ophthalmol. 2010;149(2):307-315.

14. Shin JY, Woo SJ, Yu HG, Park KH. Comparision of efficacy and safety between half-fluence and full-fluence photodynamic therapy for chronic central serous chorioretinopathy. Retina. 2011;31(1):119-126.

15. Ferris FL, Kassof A, Bresnick GH, Baily I. New visual acuity charts for clinical research. Am J Ophthalmol. 1982;94(1):91-96.

16. Forooghian F, Cukras C, Meyerle CB, Chew EY, Wong WT. Evaluation of time domain and spectral domain optical coherence tomography in the measurement of diabetic macular edema. Invest Ophthalmol Vis Sci. 2008;49(10):4290-4296.

17. Alkin Z, Ozkaya A, Agca A, et al. Early Visual and Morphologic Changes After Half-Fluence Photodynamic Therapy in Chronic Central Serous Chorioretinopathy. J Ocul Pharmacol Ther. Epub 2014 Jan 23.

18. Nicolò M, Zoli D, Musolino M, Traverso CE. Association between the efficacy of half-dose photodynamic therapy with indocyanine green angiography and optical coherence tomography findings in the treatment of central serous chorioretinopathy. Am J Ophthalmol. 2012;153(3):474-480.

\section{Dovepress}

PubMed Central and CAS, and is the official journal of The Society of Clinical Ophthalmology (SCO). The manuscript management system is completely online and includes a very quick and fair peer-review system, which is all easy to use. Visit http://www.dovepress.com/ testimonials.php to read real quotes from published authors. 